\title{
ARTICLE
}

\section{Fasciola hepatica is associated with the failure to detect bovine tuberculosis in dairy cattle}

\author{
Jen Claridge ${ }^{1}$, Peter Diggle ${ }^{1,2}$, Catherine M. McCann ${ }^{1} \uparrow$, Grace Mulcahy ${ }^{3}$, Rob Flynn ${ }^{3, \uparrow}, J_{i m ~ M c N a i r}{ }^{4}$, Sam Strain $^{4}$, \\ Michael Welsh ${ }^{4}$, Matthew Baylis ${ }^{1, \star} \&$ Diana J.L. Williams ${ }^{1, \star}$
}

Bovine tuberculosis (BTB) is a significant and intractable disease of cattle caused by Mycobacterium bovis. In the United Kingdom, despite an aggressive eradication programme, the prevalence of BTB is increasing with an unexplained, exponential rise in cases year on year. Here we show in a study involving 3,026 dairy herds in England and Wales that there is a significant negative association between exposure to the common, ubiquitous helminth parasite, Fasciola hepatica and diagnosis of BTB. The magnitude of the single intradermal comparative cervical tuberculin test used to diagnose BTB is reduced in cattle experimentally co-infected with $M$. bovis and F. hepatica. We estimate an under-ascertainment rate of about one-third ( $95 \%$ confidence interval 27-38\%) among our study farms, in the hypothetical situation of no exposure to F. hepatica. This finding may in part explain the continuing spread of BTB and the failure of the current eradication programme in the United Kingdom.

\footnotetext{
${ }^{1}$ Institute of Infection and Global Health and School of Veterinary Science, University of Liverpool, Liverpool L69 7ZJ, UK. ${ }^{2}$ Lancaster Medical School, University of Lancaster, Lancaster LA1 4YB, UK. ${ }^{3}$ School of Veterinary Medicine, University College Dublin, Belfield, Dublin 4, Ireland. ${ }^{4}$ Bacteriology Department, Agri-Food and Biosciences Institute, Veterinary Sciences Division, Stormont, Belfast BT4 3SD, UK. *These authors contributed equally to this work. †Present addresses: Division of Agriculture, Sir Arthur Lewis Community College, Morne Fortune, Castries, St Lucia, West Indies (C.M.M.); School of Veterinary Medicine and Science, University of Nottingham, LE12 5RD, UK (R.F.). Correspondence and requests for materials should be addressed to D.J.L.W. (email:williadj@liv.ac.uk).
} 
A ccurate diagnosis of an infectious disease is vital for its control, eradication and for the treatment of affected individuals. The diagnosis of many diseases requires detection of the host's immune response to the causative agent. Some pathogens, notably helminth parasites, impair their host's immune response, increasing susceptibility to infection and also affecting the sensitivity of immunologically based diagnostic tests.

Bovine tuberculosis (BTB), caused by M. bovis, is a serious disease of cattle; affected animals show loss of productivity and the economic consequences for countries with endemic BTB are exacerbated by international restrictions placed on bovine exports ${ }^{1}$. $M$. bovis is an important zoonosis; it is estimated to be responsible for $\sim 10 \%$ of human tuberculosis (TB) in Africa $^{2}$ and historically was a major public health problem in Europe, with 2,500 deaths occurring annually in the United Kingdom in the 1930s before pasteurization of milk was introduced ${ }^{3}$. BTB has been eradicated in several countries including much of the EU, Australia, Canada and most of the United States but it is still endemic in the United Kingdom, Ireland and New Zealand.

The United Kingdom has had an eradication programme in place since the 1950s, based on diagnosis using the single intradermal comparative cervical tuberculin (SICCT) test and immediate slaughter of positive animals, yet the incidence of BTB has increased exponentially in recent years. The number of new herd incidents in Great Britain in 2009 was 4,525 and government expenditure on the eradication programme was $£ 108.4$ million. The number of cattle slaughtered in 2010 was 32,411 (4,703 new herd incidents) and provisional statistics suggest an overall increase in TB incidence rate of $5.8 \%$ in 2011 compared with the same period in $2010^{4}$. By comparison, in 1999 the total cost of controlling BTB was $£ 8.2$ million with 1,666 new herd incidents recorded ${ }^{5}$. It has proved difficult to account not only for the increase in new incidents of BTB within endemic areas but also the spread of infection into new areas. Several factors have been identified as contributing to this increase such as cattle movements and reservoirs of infection within wildlife species, notably the Eurasian badger (Meles meles) ${ }^{6}$. Restrictions are now placed on cattle movements with pre-movement testing introduced in 2001, but despite this, incidence is continuing to increase with spread predominantly local but with a small number of longrange jumps ${ }^{5,7}$.

The SICCT test, approved for use in the EU and the principal test used in the United Kingdom and Ireland for ante-mortem identification of infected animals, measures a delayed type hypersensitivity response to the tuberculin antigen-purified protein derivative (PPD) and is dependent on functional antigen-specific T-cells and their capacity to secrete interferon- $\gamma($ IFN- $\gamma$ ). The diagnostic sensitivity of the SICCT test is estimated to be between $52-100 \%$ with a median value of $80 \%$ using the standard interpretation of the test. ${ }^{8}$ Several factors may account for the poor sensitivity of the SICCT test; one factor that has been considered but not fully evaluated in the field is the effect of concurrent infection with pathogens that may suppress the immune response to M. bovis ${ }^{9}$.

F. hepatica is a common trematode parasite of livestock worldwide. The prevalence of $F$. hepatica infection has increased substantially between 2000-2009 in the United Kingdom, partly due to changes in the climate that foster the development of the intermediate host and free-living stages of the parasite ${ }^{8}$, partly due to changes in land use, in particular drainage practices ${ }^{10,11}$, and partly due to the lack of drugs licensed for use in milking cattle and increasing evidence of resistance to triclabendazole ${ }^{12}$, a drug commonly used for the treatment of $F$. hepatica infection. Recent estimates indicate a herd-level prevalence of $70-80 \%$ in the UK dairy herd ${ }^{13}$. F. hepatica is known to induce an anti-inflammatory state in its host; pro-inflammatory cytokine responses are suppressed, increasing susceptibility to intracellular pathogens normally controlled by pro-inflammatory responses, such as Bordetella pertussis and
Salmonella dublin ${ }^{14,15}$. In cattle experimentally co-infected with F. hepatica and M. bovis Bacille Calmette Guerin (BCG), the SICCT test and PPD-specific IFN- $\gamma$ responses were negative in $7 / 9$ and 8/9 animals, respectively, whereas four out of five cattle infected with BCG alone were positive by both SICCT and the IFN- $\gamma$ tests ${ }^{16}$. This trend was true irrespective of whether BCG was given before or after F. hepatica. These observations led us to investigate if $F$. hepatica infection in cattle compromises the sensitivity of the SICCT test used in the United Kingdom to diagnose BTB in the field.

We show here that a significant negative association exists between exposure to F. hepatica and diagnosis of BTB, and that the magnitude of the SICCT test used to diagnose BTB is reduced in cattle experimentally co-infected with $M$. bovis and F. hepatica. These results indicate that in the presence of F. hepatica, the SICCT test used to diagnose BTB is compromised; we estimate that in our study farms, in the hypothetical situation of no exposure to F. hepatica, there is an under-ascertainment rate of about one-third (95\% confidence interval $(\mathrm{CI})=27-38 \%)$. This finding may in part explain the continuing spread of BTB and the failure of the current eradication programme in the United Kingdom.

\section{Results}

Prevalence of BTB and F. hepatica in England and Wales. Data on the prevalence of BTB and exposure to F. hepatica in 3,026 dairy herds in England and Wales were collected and the national smoothed prevalence for each pathogen calculated (Fig. 1a and b). Smoothed BTB prevalence in 2004-2007 was highest in the south and west of England and Wales (Fig. 1a). Three large clusters are apparent: northern Cornwall and Devon, the south Midlands and the southwest of Wales. A fourth, smaller and less-intense cluster is visible north of the Midlands, centred on Derbyshire. This map of BTB prevalence presents a similar picture to previous maps of BTB distribution in Great Britain calculated from all beef and dairy farms rather than, as here, a sample of dairy farms that were originally tested for exposure to F. hepatica using a bulk tank milk test ${ }^{6}$.

The smoothed F. hepatica percentage positivity (pp) value was greatest in the west of Britain (Fig. 1b). Four major clusters are the northwest of England (Cumbria), an adjoining cluster stretching south through Lancashire to the north Midlands, the northwest of Wales and a small part of southwest Wales. Some parts of Cornwall and Devon in the far southwest have moderate F. hepatica pp values.

Comparison of Fig. 1a and b shows that there is almost no overlap in the west of England and Wales in the distributions of the intense clusters of BTB prevalence and F. hepatica pp value. In the northwest of England and the northwest of Wales, F. hepatica pp values are high and BTB is of very low prevalence. In south Wales, the cluster of high $F$. hepatica pp values lies approximately between two clusters of high BTB prevalence. In the Midlands, a large area of high BTB prevalence is of low F. hepatica pp value. In Cornwall and Devon, and in the north Midlands, both BTB prevalence and F. hepatica pp values are at moderate levels. In contrast to the situation in the west of England and Wales, both BTB prevalence and F. hepatica pp values are low in most of the east of England.

Multivariable logistic regression models for BTB. One hypothesis for the strong negative spatial association between BTB and F. hepatica is that the two infections do not directly interact, but that a mutually exclusive set of environmental conditions favour BTB ${ }^{6,7}$ and $F$. hepatica ${ }^{10}$, respectively. To test this hypothesis, we developed a model for BTB, based on a previously published model that successfully predicts BTB presence or absence using a combination of environmental and farm-level variables, including animal movements between farms ${ }^{6}$. If BTB presence or absence is not affected by $F$. hepatica, then smoothed square-root-transformed $F$. hepatica 
a

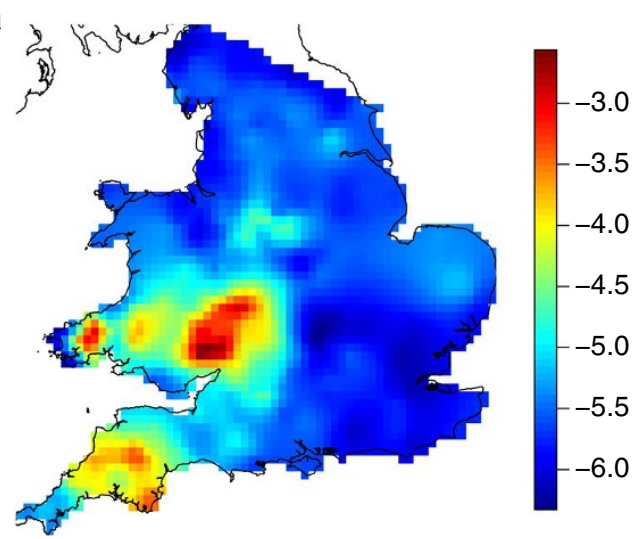

b

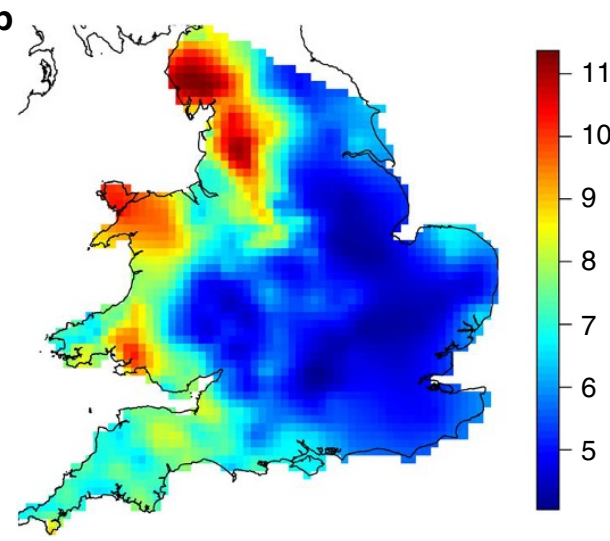

Figure 1 | Smoothed distribution of BTB and $\boldsymbol{F}$. hepatica. (a) Smoothed log-odds of an individual dairy animal having been detected as BTB-positive in 2004-2007. BTB data for 3,026 farms also tested for exposure to F. hepatica were obtained from the UK Department for Environment, Food and Rural Affairs (DEFRA) VETNET database. A data cleaning process was undertaken to ensure only records pertaining to actual livestock tests were analysed; (b) Smoothed square root of the pp value for $F$. hepatica. To determine the prevalence of exposure to $F$. hepatica, bulk milk samples were obtained from the same 3,026 dairy farms in England and Wales and tested using an antibody detection ELISA. Samples were obtained from three major milk-testing companies in England and Wales between November 2006 and January 2007.

Table 1 | Multivariable logistic regression model of BTB occurrence including smoothed square root of F. hepatica pp value.

\begin{tabular}{lccccc} 
Predictor & Coefficient & SE & P & OR & $\mathbf{L} \mathbf{9 5 \%} \mathbf{C l}$ \\
\hline Constant & -33.561 & 3.861 & $<0.001$ & & \\
Fasciola_PP & -0.486 & 0.046 & $<0.001$ & 0.61 & 0.56 \\
NN_BTB & 0.467 & 0.223 & 0.036 & 1.59 & 1.03 \\
HerdSize & 0.163 & 0.022 & $<0.001$ & 1.18 & 1.13 \\
NDVI & 0.015 & 0.002 & $<0.001$ & 1.02 & 1.01 \\
Sqrt MOff & -0.089 & 0.017 & $<0.001$ & 0.91 & 0.87 \\
MeanT & 1.196 & 0.154 & $<0.001$ & 3.31 & 1.02 \\
VPD & -2.344 & 0.352 & $<0.001$ & 0.10 & 0.95 \\
TVar & 0.071 & 0.036 & 0.048 & 1.07 & 0.05 \\
\hline
\end{tabular}

Abbreviations: Fasciola_PP, smoothed square root of F. hepatica percentage positive ELISA value; NN_BTB, nearest neighbour's smoothed BTB probability; HerdSize, square root of herd size; NDVI, normalized difference vegetation index is a measure of vegetation greenness; SqrtMOff, square root of movements off farm; MeanT, mean temperature $\left({ }^{\circ} \mathrm{C}\right)$; VPD, Vapour Pressure Deficit is a measure of lack of moisture equilibrium between an object and the atmosphere, the higher the VPD, the more rapid the rate of desiccation; TVar, temperature variance, indicates the magnitude of the seasonality, lack of moisture equilibrium between an object and the atmosphere, the higher the $\mathrm{VPD}$, the more rapid the rate of desiccation; TVar, temperature variance, indicates the magnitude of the seas
that is, larger values indicate larger differences between winter and summer extremes; OR, odds ratio; $\mathrm{L} / \mathrm{U} 95 \% \mathrm{Cl}$, lower and upper $95 \%$ confidence intervals of OR. Log-likelihood=- 953.26 .

Table 2 | Multivariable logistic regression model of BTB occurrence excluding smoothed square root of $F$. hepatica pp value.

\begin{tabular}{|c|c|c|c|c|c|c|}
\hline Predictor & Coefficient & SE & $P$ & OR & L $95 \% \mathrm{Cl}$ & U 95\% Cl \\
\hline Constant & -30.045 & 3.577 & $<0.001$ & & & \\
\hline NN_BTB & 1.020 & 0.213 & $<0.001$ & 2.77 & 1.83 & 4.21 \\
\hline HerdSize & 0.182 & 0.021 & $<0.001$ & 1.20 & 1.15 & 1.25 \\
\hline NDVI & 0.009 & 0.002 & $<0.001$ & 1.01 & 1.01 & 1.01 \\
\hline Sqrt MOff & -0.090 & 0.016 & $<0.001$ & 0.91 & 0.89 & 0.94 \\
\hline MeanT & 1.350 & 0.147 & $<0.001$ & 3.86 & 2.90 & 5.14 \\
\hline VPD & -2.443 & 0.335 & $<0.001$ & 0.09 & 0.05 & 0.17 \\
\hline TVar & 0.158 & 0.034 & $<0.001$ & 1.17 & 1.10 & 1.25 \\
\hline
\end{tabular}

pp value (our variable, Fasciola_PP) should not explain any additional variance in a statistical model for BTB driven by environmental, farm and movement variables. We therefore developed a multivariable logistic regression model for the occurrence of BTB using a sample of 1,821 farms, with model validation undertaken on the remaining subset, using proxies of the most important published variables ${ }^{6,7}$, with and without $F$. hepatica (Tables 1 and 2). In the best model excluding F. hepatica, proxies of six variables found to be significant in previous studies ${ }^{6,7}$ were highly significant (all $P<0.001$ ), as was herd size $(P<0.001)$. The risk of BTB being present on a farm increases with higher average temperature and more variable temperature and increases slightly with the normalized difference vegetation index (NDVI), but decreases with higher vapour pressure deficit (VPD). The risk increases when the nearest neighbouring farm has a higher probability of being BTB-positive and for larger herd sizes, and the risk decreases with larger numbers of movements off the farm. 
Importantly, when we add Fasciola_PP to the model, all the variables remain significant (with negligible change in coefficients and odds ratios) but Fasciola_PP has an additional, significant, negative effect on the odds of BTB being present on a farm $(O R=0.61 ; 95 \% \mathrm{CI}=0.56-$ $0.67, P<0.001$; Table 1). The model that includes Fasciola_PP fits the data significantly better than the model without that variable (difference in deviance $=126.22, P<0.001$ ). We therefore conclude that F. hepatica is an additional environmental risk factor for BTB and is negatively associated with the odds of BTB being diagnosed on a farm.

The effect of Fasciola_PP on the odds of detection of BTB, after the inclusion of environmental and farm-level variables, is robust at smaller spatial scales than in England and Wales, including both countries separately, southern England and southwest Wales.

Under-ascertainment rate of BTB in the absence of $F$. hepatica. To assess the impact of F. hepatica on BTB detection in England and Wales, we estimated the probability of farms having BTB given their environmental and farm-level characteristics, in the presence and (hypothetical) absence of F. hepatica. The results indicate that, in the absence of $F$. hepatica, the probability of farms being

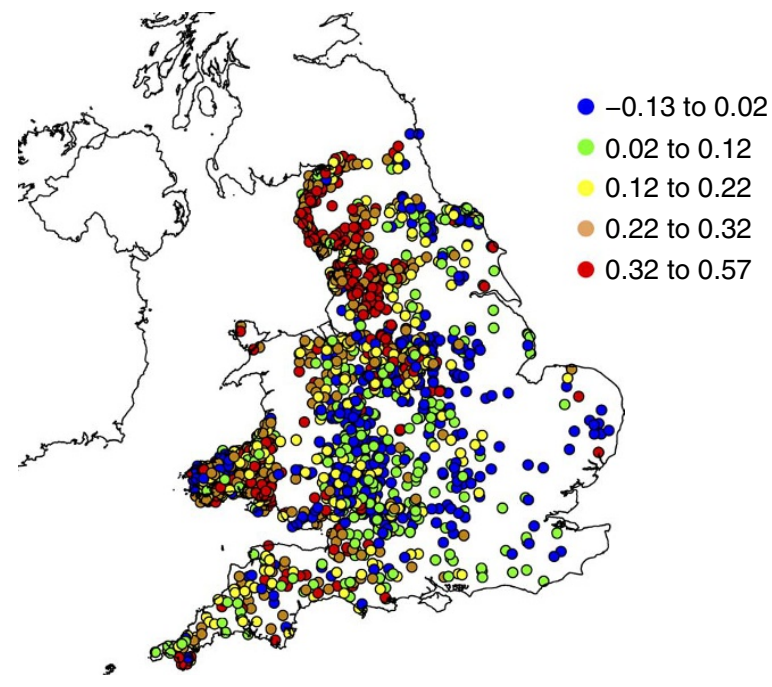

Figure 2 | Map showing the estimated effect of the presence or absence of $\boldsymbol{F}$. hepatica. Modelled probabilities of BTB in the presence or hypothetical absence of $F$. hepatica were obtained from the fitted values of the final model, with pp value either as found or set to 27 , respectively. The difference between the two probabilities ( $p p=27$ minus $p p$ as found) varied between -0.13 and +0.57 . The map shows the location of each farm, jittered randomly within a circular disc of radius $5 \mathrm{~km}$ to preserve confidentiality and colour coded by quintiles whose ranges are shown on the legend at the top-right corner of the map. diagnosed with BTB would increase substantially in several parts of England and Wales (Fig. 2). The most marked increases are in the southwest, Wales, the northern Midlands (Derbyshire), and Lancashire and Cumbria in the northwest. Using our model, we estimate that in the hypothetical absence of $F$. hepatica, the detected prevalence of BTB in our study farms would increase from 35.5 to $53 \%$, a difference of $17.5 \%$ (95\% CI, $14.5-20.4 \%$ ). This equates to an under-ascertainment rate in our study farms of about one-third $(0.175 / 0.53 ; 95 \% \mathrm{CI}=27-38 \%)$.

Experimental infection of calves with $M$. bovis and F. hepatica. The negative association between the presence of $F$. hepatica and detection of BTB could be explained by $F$. hepatica impairing the SICCT test used to diagnose BTB as there is evidence to suggest that $F$. hepatica infections reduced bystander IFN- $\gamma$ responses and compromised SICCT tests in calves infected with BCG, an avirulent strain of $\mathrm{BTB}^{16}$. To address this, six calves were experimentally infected with 150 metacercariae of $F$. hepatica, 4 weeks later they were challenged with a target dose of $5 \times 10^{2}$ colony-forming units (CFU) of a virulent strain of $M$. bovis; six calves were infected with $M$. bovis alone. SICCT tests were conducted 10 and 21 weeks after M. bovis infection (Table 3 ). The data were analysed using a linear mixed effects model fitted by maximum likelihood with week and co-infection as fixed effects and cow as a random effect. SICCT responses of co-infected cattle were significantly less than animals infected with $M$. bovis alone $(P=0.011)$, and were significantly less at week-21 post infection compared with week-10 post infection $(P=0.0013)$. The effect of co-infection did not change between 10 and 21 weeks post infection $(P=0.235)$. These results show that cattle co-infected with $F$. hepatica react less strongly to the SICCT test than those infected with $M$. bovis alone over a significant period of time.

\section{Discussion}

Here, we show a strong negative spatial association between diagnosis of BTB and exposure to F. hepatica using 3,026 dairy herds in England and Wales. When we included smoothed square-roottransformed $F$. hepatica pp value into a previously published model of BTB, which successfully predicted BTB presence or absence using a combination of environmental and farm level variables, including animal movements between farms ${ }^{6}$, the model fitted the data significantly better than the model without the presence of $F$. hepatica, confirming that the negative spatial association between BTB and F. hepatica was not associated with a mutually exclusive set of environmental conditions favouring $\mathrm{BTB}^{6,7}$ and $F$. hepatica ${ }^{10}$, respectively. This leads to the conclusion that $F$. hepatica is an additional environmental risk factor for BTB and, importantly, is negatively associated with the odds of BTB being diagnosed on a farm. These data suggest that in the presence of $F$. hepatica infection, the SICCT test is less effective, a conclusion that we corroborated by an experiment

Table 3 | SICCT test results for calves experimentally infected with either M. bovis alone or F. hepatica followed by M. bovis infection 4 weeks later.

\begin{tabular}{|c|c|c|c|c|c|}
\hline \multirow[t]{2}{*}{ Group A } & \multicolumn{2}{|c|}{$\begin{array}{l}\text { Animals co-infected with } F \text {. hepatica and } \\
\text { M. bovis (mm) }\end{array}$} & \multirow[t]{2}{*}{ Group B } & \multicolumn{2}{|c|}{$\begin{array}{c}\text { Animals infected with } M \text {. bovis } \\
\text { alone }(\mathrm{mm})\end{array}$} \\
\hline & Week 10 & Week 21 & & Week 10 & Week 21 \\
\hline 1 & 15 & 7 & 7 & 27 & 14 \\
\hline 3 & 13 & 7 & 9 & 30 & 25 \\
\hline 4 & 9 & 7 & 10 & 10 & 4 \\
\hline 5 & 21 & 14 & 11 & 25 & 18 \\
\hline
\end{tabular}


in which calves were infected with $M$. bovis alone or in combination with $F$. hepatica. The calves co-infected with $F$. hepatica reacted less strongly to the SICCT test than those infected with $M$. bovis alone when tested on two occasions at week 10 and week 21 after M. bovis infection. Previous studies have shown that cattle infected with $F$. hepatica and M. bovis BCG had significantly reduced skin thickness following standard SICCT tests, and this was associated with impaired IFN- $\gamma$ responses and enhanced IL4, IL10 and transforming growth factor (TGF)- $\beta$ secretion ${ }^{16}$. This concurs with murine models in which $F$. hepatica or specific $F$. hepatica antigens have been shown to modulate dendritic cell and macrophage function, leading to the induction of regulatory T-cell populations expressing TGF- $\beta$ and IL10, which in turn inhibit bystander and auto-antigenspecific Th1 and Th17 responses and release of inflammatory mediators such as IFN- $\gamma$ and IL12 $2^{14,17-20}$. Similarly in humans, helminth infections suppress T-cell and IFN- $\gamma$ responses to PPD and reduce the efficacy of BCG vaccination ${ }^{21-24}$. These data are consistent with the conclusion from our findings that in the presence of $F$. hepatica infection, the efficacy of the SICCT test used to diagnose BTB is compromised.

Although the magnitude of the response to the SICCT test in the co-infected calves was significantly less than that in calves infected with $M$. bovis alone, all six co-infected animals had responses that would be considered positive under field conditions (comparative increase in skin thickness of more than $4 \mathrm{~mm}$ ), nevertheless the mean difference in skin thickness was $42 \%$ less than in those infected with $M$. bovis alone. It was not feasible to replicate under experimental conditions the situation in the field where animals are likely to have higher F. hepatica burdens and we have not yet explored the interaction between intensity and relative timing of each infection and the impact each may have on the SICCT. But it is plausible that a F. hepatica infection has the effect of pushing weak SICCT positives into a negative classification in the field where interpretation of the test is not always clear cut.

An alternative explanation for both our epidemiological analysis and the results from the experimental infections is that $F$. hepatica reduces the susceptibility of cattle to BTB. In a study in which 200 in-contact and 200 cattle classified as BTB-infected by SICCT (that is, reactors) were investigated in depth, a consistent negative association was described between having antibodies to F. hepatica and having confirmed BTB, in both groups ${ }^{25}$. Another study ${ }^{26}$ showed that experimentally co-infected cattle had fewer BTB lesions at slaughter and fewer tissue samples that were culture positive for $M$. bovis although these differences were not shown to be significant. Moreover, neither of these studies investigated whether co-infection influenced the nature, development or distribution of lesions and bacterial spread, which could also explain these results. Also both the SICCT and the PPD-specific IFN- $\gamma$ response were suppressed in BCG/F. hepatica co-infected calves and this trend was true irrespective of whether BCG was given before or after $F$. hepatica ${ }^{16}$ suggesting overall that the most likely interpretation of our findings is that a proportion of BTB-positive animals are being missed by the SICCT test.

If, as our data suggest, $F$. hepatica suppresses the SICCT response of BTB-infected cattle, our model indicates a substantial degree of under-ascertainment of BTB using the SICCT test. Undetected cases have the potential to spread infection to other cattle within the herd, to wildlife within the area and, if moved, to herds in different parts of the country. F. hepatica is a ubiquitous parasite, whose prevalence is predicted to fluctuate considerably in areas of the United Kingdom over the next 50 years due to changes in climate ${ }^{8}$. It is possible that treatment of cattle with flukicidal drugs, before testing, might increase the detection rate of BTB, leading in the short term to an apparent surge in incidence, but, in the longer term, improved control of the disease. Studies in helminth-infected humans suggest that vaccine responses to BCG are compromised ${ }^{24}$; extrapolating these findings to cattle, infection with $F$. hepatica may compromise induction of vaccine responses to M. bovis, an important consideration in view of the current intention to develop and apply vaccines to cattle to help control the BTB epidemic in the United Kingdom and Ireland. This is the first wide-scale analysis of the interaction between F. hepatica and M. bovis and provides direct evidence of a significant negative association between the two infections, the most likely explanation for which is the impact of $F$. hepatica on the host's immune system reducing the sensitivity of the principle diagnostic test used for M. bovis. This finding has significant implications for the UK and Ireland's BTB eradication programmes.

\section{Methods}

F. hepatica data. Bulk milk samples from 3,026 dairy farms in the England and Wales ${ }^{13}$ were tested using an antibody detection enzyme-linked immunosorbent assay (ELISA) ${ }^{27}$. The test result was expressed as the pp value, which is the ratio of the optical density reading for the test sample and the optical density reading for a positive control sample. A cutoff pp value of 27 was used to define a positive herd; at this cutoff the diagnostic sensitivity of the test is $96 \%(95 \% \mathrm{CI}=89-100 \%)$ and the diagnostic specificity is $80 \%(95 \% \mathrm{CI}=66-94 \%)^{27}$.

BTB data. BTB data was obtained for each of the 3,026 farms. Two sources of uncertainty are acknowledged in the BTB data. First, SICCT test sensitivity and specificity are imperfect, leading to false-positive and false-negative results. Positive skin test results are normally confirmed if typical $M$. bovis lesions are found at post mortem examination or if positive culture for M. bovis is obtained. Owing to the low sensitivity of laboratory culture and the frequent absence of visible lesions, the number of animals reacting to the skin test was used to denote farms with BTB breakdowns, rather than the number of confirmed cases of BTB. Second, BTB testing occurs at different frequencies according to parish prevalence of BTB. BTB testing intervals range from every 1 to every 4 years routinely, but are also conducted when necessary for pre-movement testing, providing the farm has not been tested in the last 60 days; testing is repeated 60 days later if a reactor animal is detected. Once a herd is free from reactors it must test negative to two subsequent whole-herd tests, 60 days apart, to be considered 'Officially TB free'. We analysed the cumulative incidence of BTB in the 3,026 farms from 1 January 2004 to 31 December 2007; this 4-year period was chosen to, first, ensure that all farms had been tested for BTB at least once and, second, include the period when the same farms were tested for F. hepatica.

Smoothing of F. hepatica pp value and BTB data. Smoothing is a statistical process that aims to capture important patterns in data while reducing noise. We used model-based geostatistics ${ }^{28}$ to smooth the two sets of disease data. First, the 3,026 farms were randomly allocated to 1,984 to be used for model development (designated as analysis locations), while the remaining 1,042 (hold-out locations) were reserved for model validation. The underlying statistical model is that $Y_{i}=S\left(x_{i}\right)+Z_{i}: i=1, n$, where $Y_{i}$ is the observed value of a response variable at location $x_{i}, S(x)$ is an unobserved spatially varying surface that is modelled as the realization of a spatial stochastic process and $Z_{i}$ is an independent random perturbation with variance $\hat{o}^{2}$ that accounts for sampling variation and/or micro-scale spatial variation in the immediate vicinity of $x_{i}$ ('immediate' meaning, roughly, less than the smallest distance between any two data locations). The properties of the stochastic process $S(\cdot)$ are encapsulated in its mean, its variance, $\sigma^{2}$ and the covariance, $\sigma^{2} \rho(\mu / \varphi)$ between values of $S(x)$ at a pair of locations a distance $\mu$ apart, where $\varphi$ is a parameter representing the rate at which the spatial correlation decays with increasing distance. For F. hepatica, we used as the response variable squareroot-transformed pp value, while for BTB we used the log-odds of prevalence.

The theoretical variogram of $Y$ is the function $V(\mu)=\tau^{2}+\sigma^{2}(1-\rho(\mu / \varphi))$. We specified the parametric model $\rho(\mu / \varphi)=\exp (-\mu / \varphi)$ by inspecting the sample variogram of the data, estimated the model parameters, $\mu, \sigma^{2}, \varphi$ and $\tau^{2}$ by the method of maximum likelihood and used the fitted model to compute the minimum mean square error predictor of $S(x)$ at each of the 1,984 data locations, at each of the 1,042 hold-out locations and on a regular grid spanning England and Wales at $5 \times 5 \mathrm{~km}$ resolution so as to generate a national map of the predicted spatial variation in $S(x)$. The variograms for BTB and Fasciola_PP value are shown in Fig. 3.

The analysis was conducted using the R open-source software environment (www.r-project.org).

Covariate data. Covariate data were obtained for published descriptions of explanatory variables for $\mathrm{BTB}^{6}$, or close proxies of them. We derived environmental variables from both meteorological and satellite-derived data. Meteorological variables, including maximum and minimum monthly temperatures, and vapour pressure (VP) were obtained for the period 2001-2005, from the UK's Meteorological Office as raster maps with a $5 \times 5 \mathrm{~km}$ resolution. For each variable, data for each farm location were extracted from the raster layer using ArcGIS v 9.3 (ArcMap version 9.3, ESRI, USA). In brief, the raster layer was fitted to the base map containing point data on farm location, using the Georeferencing tool bar. The raster layer 
was then stretched to fit the underlying base map closely in $\sim 50$ positions around the coastline of the base map. The transformation option was highlighted from the georeferencing menu, and a first-order transformation was decided as most acceptable. The root mean square of the transformation should be less than or equal to the pixel size of the data set when checked. Point data pertaining to each test was added to the base map and values of the overlying raster layer were extracted to the attributes table for each newly created layer contained in a 'variable point' column that contained the newly extracted raster data as point data. Microsoft Access 2007 was then used to assimilate a table with each farm location and each raster layer value for that farm. The average temperature for each farm was then calculated from the maximum and minimum temperatures extracted. This method produces smoother values with fewer high outliers.

VPD was calculated ${ }^{29}: \mathrm{VPD}(\mathrm{mb})=\mathrm{SVP}-\mathrm{VP}$, where $\mathrm{SVP}=$ saturation vapour deficit $(\mathrm{mb}), \mathrm{VP}=$ vapour pressure $(\mathrm{mb})$ and $\log _{10}(\mathrm{SVP})=9.24349-(2305 / T)-$ $\left(500 / T^{2}\right)-\left(100000 / T^{3}\right)$, where $T=$ mean monthly temperature $(\mathrm{K})$.
Moderate-resolution imaging spectroradiometer (MODIS) imagery data for normalized difference vegetation index (NDVI) and land surface temperature were obtained from the NASA Terra satellite ${ }^{30}$

NDVI was obtained for each farm location. Bidirectional reflectance distribution function-adjusted reflectance was initially recorded and from this NDVI was extracted $^{31}$

The temperature variance ( $T$ Var) was calculated using the variance in temperature of all months and years (January 2001-December 2005 inclusive).

Other covariates were derived as follows. Herd size was obtained from the VETNET database managed by the Animal Health and Veterinary Laboratories Agency (AHVLA), Weybridge. We used proxies of the two remaining variables. Instead of movements onto the farm from infected areas only ${ }^{6}$, we used the total number of movements of cattle (from any area) onto and off each farm during the 4-year period; assuming more cattle movements per se may indicate an increased risk of the acquisition of BTB-infected stock. Farm-level animal movement data for
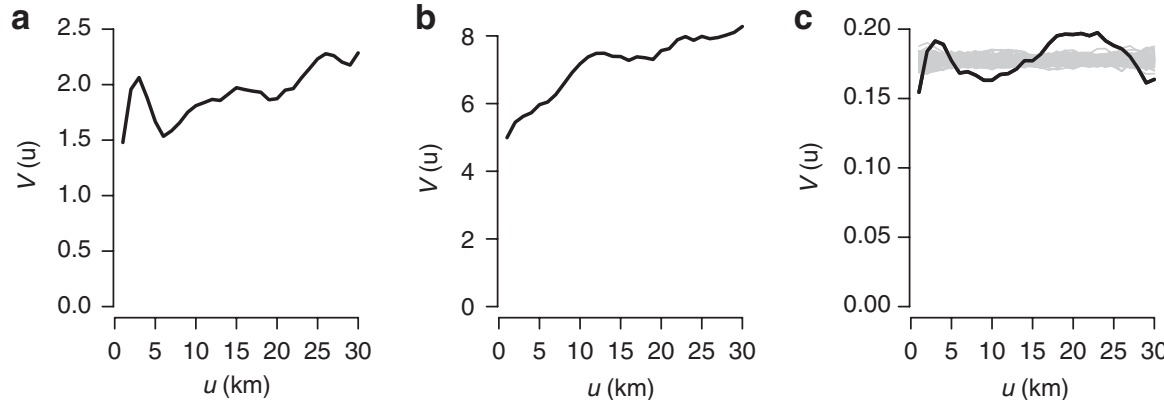

Figure 3 | Empirical variograms for incidence of BTB, $\boldsymbol{F}$. hepatica infection and the residuals from the fitted model. (a) Log-odds of the herd-level cumulative incidence of BTB; (b) square-root of the pp value; and (c) the residuals from the fitted model; the grey lines are empirical variograms computed from 99 independent permutations of the residuals over the locations. These provide an informal tolerance envelope for the empirical variogram when the residuals are spatially independent. A formal Monte Carlo test rejects the spatial independence hypothesis $(P=0.01)$ but the absence of a clear rising trend in the empirical variogram, in contrast to the behaviour of the other two variograms, suggests that the residual spatial correlation is relatively weak. The $x$ axis, $u$, denotes distance in kilometres; the $y$ axis, $V(u)$, denotes the estimated value of the variogram at distance $u$.

Table 4 | Complete list of variables available for multivariable logistic regression analysis.

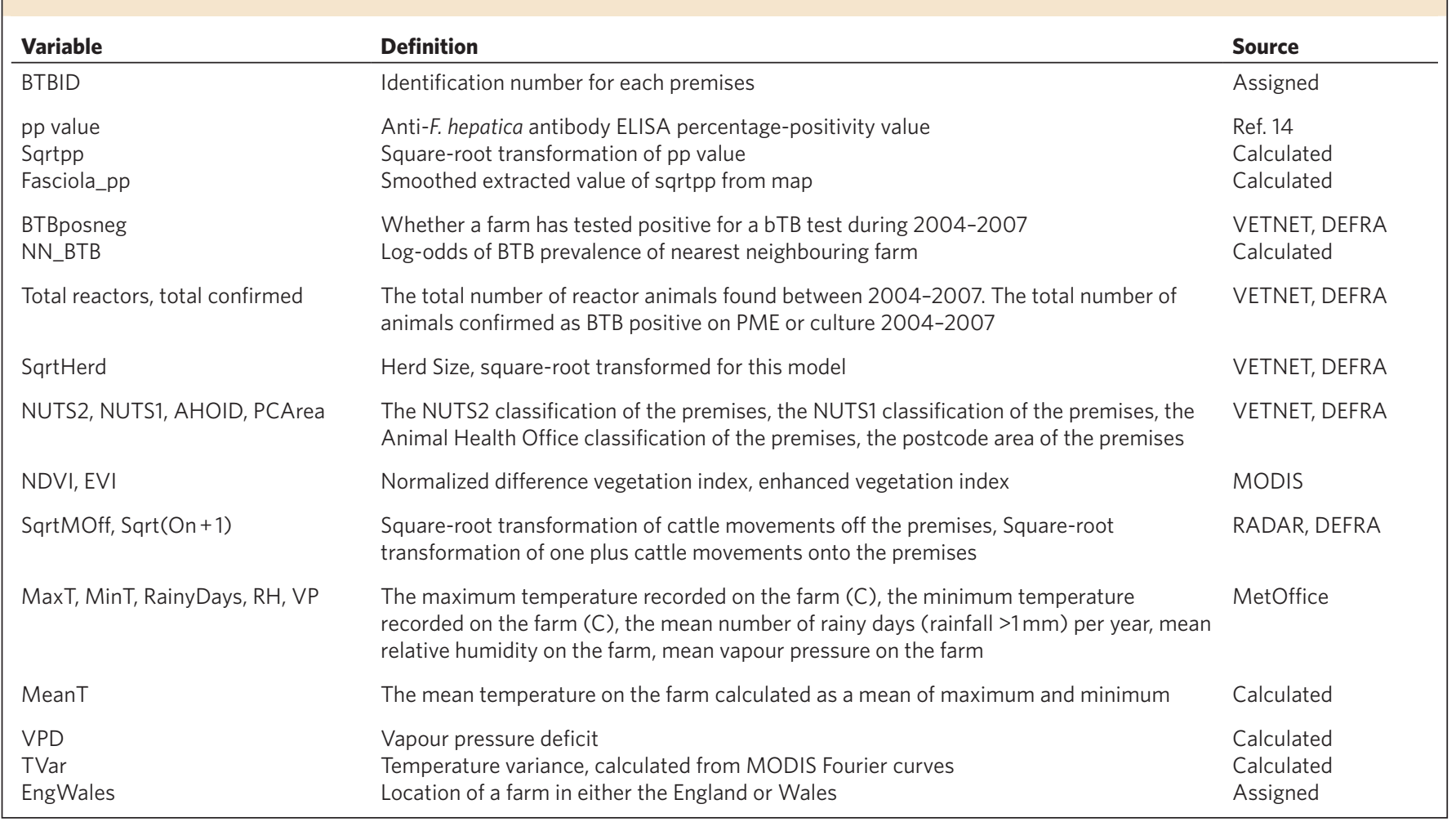


2004-2007 were obtained from DEFRA (Rapid Analysis and Detection of Animalrelated Risk). Finally, we obtained the log-odds of prevalence of BTB of the nearest neighbour, from our analysis farms, using the Hawth's Analysis Tools extension (version 3.27, www.spatialecology.com/htools/download.php) in ArcMap.

Data for herd size and movements on and off premises were square root transformed to normalize their distributions.

Multivariable logistic regression model. A BTB-positive farm was defined as a premises having at least one animal reacting positively to the BTB SICCT test in the 4 -year period. The association between BTB and the explanatory variables was explored using binary logistic regression analysis (Minitab 16). Significance was determined at the $5 \%$ level.

Twenty-five variables (Table 4 ) were initially available and tested in the logistic regression model. Variables were removed individually by backward elimination, beginning with the variable with the least significant $P$-value. This approach allowed any changes towards significance to be monitored with every variable removed. The final model contained only variables that were statistically significant $(P<0.05)$ and where the confidence limits around the adjusted odds ratio did not include 1 . Stepwise regression and forward regression models produced the same final model.

Initially, 3,026 farms were included in the $F$. hepatica ELISA dataset. Four pairs of farms had different county-parish-holding $(\mathrm{CPH})$ numbers but shared the same co-ordinates. Data were missing from 224 farms due to incomplete coverage by raster layers. This left a total sample size of 2,794 farms for regression analysis. These were randomly divided into two sets: 1,821 analysis farms, for model development, and 973 hold-out farms, for model validation.

A variogram of the residuals of the logistic regression model (Fig. 3) indicated some remaining but weak, unexplained spatial correlation, decaying from $\sim 0.2$ to zero over the range $0-30 \mathrm{~km}$.

Experimental infections. All procedures were conducted under the Animals (Scientific Procedures) Act, 1986 and animals were housed in a containment level 3 facility for the duration of the experiment. Twelve castrated male Friesian calves aged between 17 and 21 weeks of age were used; six were infected with a single oral dose of $150 \mathrm{~F}$. hepatica metacercariae of the Cullompton isolate (Ridgeway Research Ltd. UK). Four weeks later, all 12 calves were infected with $M$. bovis (isolate AF2122/99, kindly provided by Dr Martin Vordermeier, AHVLA, Weybridge) by exposure to an airstream carrying aerosolized mycobacteria using a Madison chamber modified for use with cattle. During the 3-min infection period when aerosolized M. bovis was delivered to each animal individually, air inspired by each calf was monitored using a pneumotachometer and individual airstream samples were taken into all-glass impingers containing phosphate buffer solution to calculate the infection dose delivered to each animal. The intended target infection dose was $500 \mathrm{CFU}$ of $M$. bovis. The mean dose of $M$. bovis actually delivered was calculated as $506 \mathrm{CFU}$ (range 271-683, s.d. 182) for the M. bovis only group and 548 CFU (range 342-674, s.d. 129) for the co-infection group. There was no statistical difference between the mean values for each group. The SICCT test was carried out 10 and 21 weeks post $M$. bovis infection in accordance with the EU and UK regulations. The tuberculins PPDa and PPDb were injected into two intradermal sites that had been clipped and measured for skin thickness using callipers. Each injection site was checked to ensure intradermal delivery. At $72 \mathrm{~h}$ post injection, the skin thickness of each site was measured and recorded. The same person performed all injections and measurements to limit operator variation. The data were analysed using a linear mixed effects model fitted by maximum likelihood with week and co-infection as fixed effects and cow as a random effect, as implemented in the $\mathrm{R}$ open-source software environment (www.r-project.org)

\section{References}

1. Gordon, S. V. Bovine TB: stopping disease control would block all live exports. Nature 456, 700 (2008).

2. Cosivi, O. et al. Zoonotic tuberculosis due to Mycobacterium bovis in developing countries. Emerg. Infect. Dis. 4, 59-70 (1998).

3. de la Rua-Domenech, R. Human Mycobacterium bovis infection in the United Kingdom: Incidence, risks, control measures and review of the zoonotic aspects of bovine tuberculosis. Tuberculosis (Edinb) 86, 77-109 (2006).

4. DEFRA. http://archive.defra.gov.uk/foodfarm/farmanimal/diseases/atoz/tb/ stats/county.htm (2011).

5. DEFRA. Bovine tuberculosis infection status in cattle: Mid year surveillance report July 2009-June 2010, http://archive.defra.gov.uk/foodfarm/farmanimal/ diseases/atoz/tb/documents/btb-surv-report.pdf (2010).

6. Gilbert, M. et al. Cattle movements and bovine tuberculosis in Great Britain. Nature 435, 491-496 (2005).

7. Wint, G. R. et al. Mapping bovine tuberculosis in Great Britain using environmental data. Trends Microbiol. 10, 441-444 (2002).

8. Fox, N. J. et al. Predicting impacts of climate change on Fasciola hepatica risk. PLoS One 6, e16126 (2011).

9. de la Rua-Domenech, R. et al. Ante mortem diagnosis of tuberculosis in cattle a review of the tuberculin tests, gamma-interferon assay and other ancillary diagnostic techniques. Res. Vet. Sci. 81, 190-210 (2006).
10. McCann, C. M., Baylis, M. \& Williams, D. J. The development of linear regression models using environmental variables to explain the spatial distribution of Fasciola hepatica infection in dairy herds in England and Wales. Int. J. Parasitol. 40, 1021-1028 (2010).

11. Bennema, S. C. et al. Relative importance of management, meteorological and environmental factors in the spatial distribution of Fasciola hepatica in dairy cattle in a temperate climate zone. Int. J. Parasitol. 41, 225-233 (2011).

12. Brennan, G. P. et al. Understanding triclabendazole resistance. Exp. Mol. Pathol. 82, 104-109 (2007)

13. McCann, C. M., Baylis, M. \& Williams, D. J. Seroprevalence and spatial distribution of Fasciola hepatica-infected dairy herds in England and Wales. Vet. Rec. 166, 612-617 (2010)

14. Brady, M. T., O’Neill, S. M., Dalton, J. P. \& Mills, K. H. Fasciola hepatica suppresses a protective Thl response against Bordetella pertussis. Infect. Immun. 67, 5372-5378 (1999)

15. Aitken, M. M., Hughes, D. L., Jones, P. W., Hall, G. A. \& Smith, G. S. Immunological responses of fluke-infected and fluke-free cattle to Salmonella dublin and other antigens. Res. Vet. Sci. 27, 306-312 (1979).

16. Flynn, R. J., Mannion, C., Golden, O., Hacariz, O. \& Mulcahy, G. Experimental Fasciola hepatica infection alters responses to tests used for diagnosis of bovine tuberculosis. Infect. Immun. 75, 1373-1381 (2007).

17. Hamilton, C. M. et al. The Fasciola hepatica tegumental antigen suppresses dendritic cell maturation and function. Infect. Immun. 77, 2488-2498 (2009).

18. Dowling, D. J. et al. Major secretory antigens of the helminth Fasciola hepatica activate a suppressive dendritic cell phenotype that attenuates Th17 cells but fails to activate Th2 immune responses. Infect. Immun. 78, 793-801 (2010).

19. Donnelly, S. et al. Helminth cysteine proteases inhibit TRIF-dependent activation of macrophages via degradation of TLR3. J. Biol. Chem. 285, 3383-3392 (2010).

20. Walsh, K. P., Brady, M. T., Finlay, C. M., Boon, L. \& Mills, K. H. Infection with a helminth parasite attenuates autoimmunity through TGF-beta-mediated suppression of Th17 and Th1 responses. J. Immunol. 183, 1577-1586 (2009).

21. Babu, S. et al. Attenuation of toll-like receptor expression and function in latent tuberculosis by coexistent filarial infection with restoration following antifilarial chemotherapy. PLoS Negl. Trop. Dis. 3, e489 (2009).

22. Wammes, L. J. et al. Regulatory T cells in human geohelminth infection suppress immune responses to BCG and Plasmodium falciparum. Eur. J. Immunol. 40, 437-442 (2010)

23. Elias, D. et al. Effect of deworming on human $\mathrm{T}$ cell responses to mycobacterial antigens in helminth-exposed individuals before and after bacille CalmetteGuerin (BCG) vaccination. Clin. Exp. Immunol. 123, 219-225 (2001).

24. Elias, D., Britton, S., Aseffa, A., Engers, H. \& Akuffo, H. Poor immunogenicity of BCG in helminth infected population is associated with increased in vitro TGF-beta production. Vaccine 26, 3897-3902 (2008).

25. DEFRA. Pathogenesis and diagnosis of tuberculosis in cattle - complementary field studies Report No. SE3013, http://randd.defra.gov.uk/Default.aspx?Menu= Menu\&Module $=$ More\&Location $=$ None\&ProjectID=9317 (2005).

26. Flynn, R. J. et al. Co-Infection of cattle with Fasciola hepatica and Mycobacterium bovis- immunological consequences. Transbound. Emerg. Dis. 56, 269-274 (2009).

27. Salimi-Bejestani, M. R. et al. Prevalence of Fasciola hepatica in dairy herds in England and Wales measured with an ELISA applied to bulk-tank milk. Vet. Rec. 156, 729-731 (2005).

28. Diggle, P. \& Ribeiro, P. Model-Based Geo-Statistics (Springer, 2006)

29. Hay, S. I. \& Lennon, J. J. Deriving meteorological variables across Africa for the study and control of vector-borne disease: a comparison of remote sensing and spatial interpolation of climate. Trop. Med. Int. Health 4, 58-71 (1999).

30. Scharlemann, J. P. et al. Global data for ecology and epidemiology: a novel algorithm for temporal Fourier processing MODIS data. PLoS One 3, e1408 (2008).

31. Jenkins, G., Perry, M. \& Prior, J. The Climate Of The United Kingdom and Recent Trends 1-25 (UKCIP08, Hadley Centre, Met Office: Exeter, 2007).

\section{Acknowledgements}

We are grateful to Georgette Kluiters, Cyril Caminade and Jon Read for help with data extraction and analysis; Christian Setzkorn for provision of cattle movement data; and William Wint and David Rogers for MODIS data. We thank Andy Mitchell, AHVLA, for provision of BTB data and for permission to use cattle movement data. We wish to thank Jane Rees for reviewing the manuscript. This work was funded by a BBSRC DTG award to D.J.L.W. and M.B., and an EU project grant - DELIVER (Contract no: FOOD-CT2004-023025) awarded to D.J.L.W.

\section{Author contributions}

J.C.: model development, data processing. P.D.: statistical analysis and smoothing methodology. C.M.Mc.: collection of milk samples and testing for antibodies to F. hepatica. G.M., R.F., J.M., S.S., M.W.: experimental infection of calves with $M$. bovis and F. hepatica, immunological analysis, SICCT determination, post mortem 
examinations and bacterial analysis. M.B., D.J.L.W.: conception of project, project design. The manuscript was written by J.C., M.B. and D.J.L.W. but all authors contributed.

\section{Additional information}

Competing financial interests: The authors declare no competing financial interests.

Reprints and permission information is available online at http://npg.nature.com/ reprintsandpermissions/
How to cite this article: Claridge, J. et al. Fasciola hepatica is associated with the failure to detect bovine tuberculosis in dairy cattle. Nat. Commun. 3:853 doi: 10.1038/ncomms1840 (2012).

License: This work is licensed under a Creative Commons Attribution-NonCommercialShare Alike 3.0 Unported License. To view a copy of this license, visit http:// creativecommons.org/licenses/by-nc-sa/3.0/ 